\title{
HS 2237+8154: A NEW PRE-CV JUST ABOVE THE PERIOD GAP
}

\author{
B. T. Gänsicke, ${ }^{1,2}$ S. Araujo-Betancor, ${ }^{3.2}$ H.-J. Hagen, ${ }^{4}$ E. T. Harlaftis, ${ }^{5}$ S. Kit iionlis. ${ }^{j}$ S. Dreizler, ${ }^{6}$ \\ and D. Engels ${ }^{4}$
}

\begin{abstract}
We report follow-up observations of the new pre-cataclysmic variable HS $2237+8154$, identified from the Hamburg Quasar Survey. The orbital period derived from ellipsoidal modulation observed in the $R$-band as well as from the $\mathrm{H}_{\alpha}$ radial velocity variation is orb $=$ $\mathbf{1 7 8 . 1 0} \pm 0.08 \mathrm{~min}$. We briefly discuss the evolutionary state of this system.
\end{abstract}

We have identified a detached white dwarf/red dwarf binary, HS $2237+8154$, in the course of our studies of the stellar content of the Hamburg Quasar Survey (e.g. Gänsicke et al. 2000; Gänsicke et al. 2002; Araujo-Betancor et al. 2003). Time-resolved follow-up observations of HS $2237+8154$ were carried out at the $2.5 \mathrm{~m}$ Isaac Newton Telescope in August/September 2002, and differential $R$-band photometry was obtained in September/October 2002 using the $1.2 \mathrm{~m}$ Kryoneri telescope.

The spectrum of HS $2237+8154$ (Fig. 1) clearly reveals the composite nature of the object, with prominent $\mathrm{TiO}$ absorption bands in the red part of the spectrum, hallmarks of a late type star, and broad Balmer absorption lines in the blue part of the spectrum, characteristic of the high-gravity atmosphere of a white dwarf. Narrow emission of $\mathrm{H}_{\alpha}$ and $\mathrm{H}_{\beta}$ is detected, which is most likely associated with chromospheric activity on the latetype companion. We have fitted the spectrum of HS $2237+8154$ with a two-component consisting of synthetic white dwarf spectra with a fixed surface gravity $(\log g=8)$ and a library of observed M-dwarf templates. The best fit is found for a white dwarf with $T_{\text {eff }}=11500 \pm 1500 \mathrm{~K}$ and a late-type companion with a spectral type dM $3.5 \pm 0.5$ (Fig. 1). From the scaling factors between the model/template spectra and the observed spectrum of HS $2237+8154$ we derive a distance of $d=105 \pm 25 \mathrm{pc}$.

The $R$ band photometry shows a quasi-sinusoidal variation with a period of $\sim 90 \mathrm{~min}$ and a peak-topeak amplitude of $\sim 0.1 \mathrm{mag}$. There is little evidence

\footnotetext{
${ }^{1}$ University of Warwick, Coventry CV4 7AL, LK.

${ }^{2}$ University of Southampton, UK

${ }^{3}$ Space Telescope Science Institute, USA.

${ }^{4}$ Hamburger Sternwarte, Germany.

${ }^{5}$ National Observatory of Athens, Greece.

${ }^{6}$ Universitäts-Sternwarte Göttingen, Germany.
}

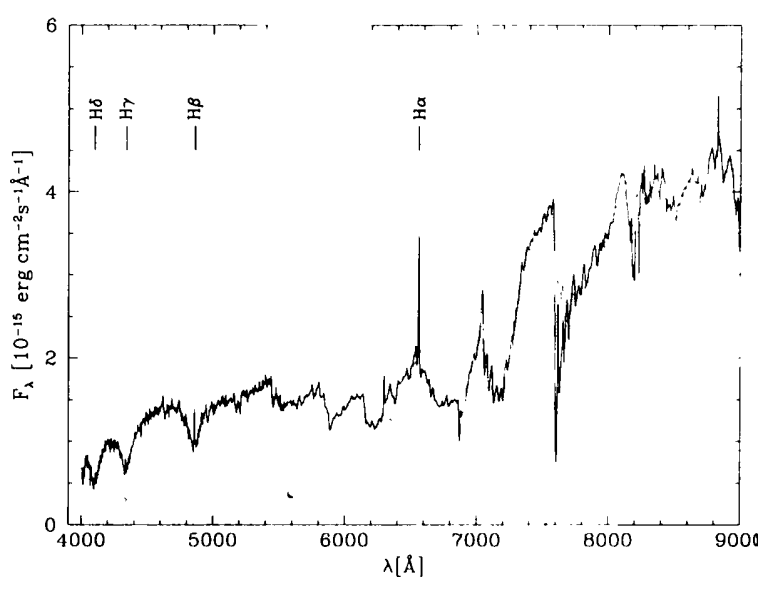

Fig. 1. The Calar Alto TWIN idenfication spectrum of HS 2237+8154. Plotted in graty is a two-component fit consisting of a white dwarf model $\left(T_{\text {eff }}=12000 \mathrm{~K}\right.$, $\log g=8.0$ ) and a dM3.5 template (Gl273).

for reflection effect in the $R$ band light curve, which is not too surprising given the low temperature of the white dwarf. We therefore interpret the observed photometric variation as ellipsoidal modulation. A detailed period analysis results in $P_{\text {orb }}=2 \times P_{\text {phot }}=$ $178.10 \mathrm{~min}$. This period is confirmed by the analysis of the $\mathrm{H}_{r \mathrm{r}}$ radial velocity variation, determined from single Gaussian fits to the observed emission lines. Combining the constraints obtained from the radial velocity of the secondary and from the ellipsoidal modulation, we derive a binary inclination of $i \simeq$ $50^{\circ}-70^{\circ}$ and stellar masses of $M_{\mathrm{wd}}=0.47-0.67 \mathrm{M}$ : and $M_{\text {sec }}=0.2-0.4 M_{\text {(.). }}$.

All observations imply that the secondary star must be nearly Roche-lobe: filling. Consequently, HS 2237+8154 may be either a pre-cataclysmic variable close to the start of mass transfer, or - considering its orbital period - a cataclysmic variable that terminated mass transfer and entered the period gap. or a hibernating nova.

\section{REFEREN('ES}

Araujo-Betancor, S., Gänsicke, B. T., Hagen, H.-J., Rodriguez-Gil, P., \& Engels, D. 2003, A\& A. 406. 213 Gänsicke, B. T., Fried, R. E., Hagen, H.-J., et al. 2000. A\&A, 356, L79

Gänsicke, B. T., Hagen, H. J., \& Engels, D. 2002, in The Physics of Cataclysmic Variables and Related $\mathrm{Ob}-$ jects, ed. B. 'T. Gänsicke, K. Beuermann, \& K. Reinsch (ASP Conf. Ser. 261), 190 199 